\title{
The neural signatures of distinct psychopathic traits
}

\author{
Justin M. Carré ${ }^{1,2}$, Luke W. Hyde ${ }^{3}$, Craig S. Neumann ${ }^{4}$, Essi Viding ${ }^{5}$, \\ and Ahmad R. Hariri ${ }^{1,6}$ \\ ${ }^{1}$ Laboratory of NeuroGenetics, Department of Psychology and Neuroscience, Duke University, \\ Durham, NC, USA \\ ${ }^{2}$ Department of Psychology, Wayne State University, Detroit, MI, USA \\ ${ }^{3}$ Department of Psychology, Center for the Neural Basis of Cognition, University of Pittsburgh, \\ Pittsburgh, PA, USA \\ ${ }^{4}$ Department of Psychology, University of North Texas, Denton, TX, USA \\ ${ }^{5}$ Division of Psychology and Language Sciences, University College London, London, UK \\ ${ }^{6}$ Institute for Genome Sciences and Policy, Duke University, Durham, NC, USA
}

\begin{abstract}
Recent studies suggest that psychopathy may be associated with dysfunction in the neural circuitry supporting both threat- and reward-related processes. However, these studies have involved small samples and often focused on extreme groups. Thus, it is unclear to what extent current findings may generalize to psychopathic traits in the general population. Furthermore, no studies have systematically and simultaneously assessed associations between distinct psychopathy facets and both threat- and reward-related brain function in the same sample of participants. Here, we examined the relationship between threat-related amygdala reactivity and reward-related ventral striatum (VS) reactivity and variation in four facets of self-reported psychopathy in a sample of 200 young adults. Path models indicated that amygdala reactivity to fearful facial expressions is negatively associated with the interpersonal facet of psychopathy, whereas amygdala reactivity to angry facial expressions is positively associated with the lifestyle facet. Furthermore, these models revealed that differential VS reactivity to positive versus negative feedback is negatively associated with the lifestyle facet. There was suggestive evidence for gender-specific patterns of association between brain function and psychopathy facets. Our findings are the first to document differential associations between both threat- and reward-related neural processes and distinct facets of psychopathy and thus provide a more comprehensive picture of the pattern of neural vulnerabilities that may predispose to maladaptive outcomes associated with psychopathy.
\end{abstract}

Keywords: Psychopathic traits; Threat; Reward; Amygdala; Ventral striatum.

Psychopathy is a personality construct characterized by superficial charm, shallow emotions, lack of empathy, lack of guilt or remorse, irresponsibility, impulsivity, deceitfulness, and persistent antisocial behaviors (Hare, 2006). Although psychopaths represent approximately $1-2 \%$ of the general population (Hare, 1998; Neumann \& Hare, 2008), they commit more than $30 \%$ of violent crimes, costing society an estimated \$250-400 billion dollars each year (Kiehl
\& Buckholtz, 2010). Psychopaths demonstrate deficits in fear conditioning, stimulus-reinforcement learning, and processing facial expressions of fear and sadness, which together may contribute to persistent antisocial behavior (Blair, 2007).

The amygdala, one key component of the neural circuitry mediating the above processes (Davis \& Whalen, 2001), has received considerable attention in psychopathy research. Specifically, neuroimaging

Correspondence should be addressed to: Justin M. Carré, Ph.D., Department of Psychology, Wayne State University, 5057 Woodward Ave., Detroit, MI, USA. E-mail: justin.carre@wayne.edu 
studies have revealed that criminal psychopaths have decreased amygdala reactivity during fear conditioning (Birbaumer et al., 2005) and an emotional memory task (Kiehl et al., 2001), as well as when viewing pictures depicting moral violations (Harenski, Harenski, Shane, \& Kiehl, 2010). Consistent with these findings in adults, children and adolescents with conduct problems and callous-unemotional traits, characteristics considered precursors to the development of psychopathy, demonstrate relatively decreased amygdala reactivity to fearful facial expressions (Jones, Laurens, Herba, Barker, \& Viding, 2009; Marsh et al., 2008). These latter findings suggest that amygdala dysfunction is apparent relatively early in development and may contribute to the development of psychopathy in adulthood.

Although psychopathy has historically been viewed as a categorical construct (i.e., a person is or is not a psychopath), recent work suggests that psychopathy may be better conceptualized as a constellation of personality and behavioral traits that vary along a continuum (Hare \& Neumann, 2005, 2008). Psychometric evidence from forensic and community samples (Hill, Neumann, \& Rogers, 2004; Neumann \& Hare, 2008) indicates that psychopathic traits vary continuously along four facets: (1) interpersonal (e.g., conning, manipulative, deceitful), (2) affective (e.g., coldheartedness, lack of guilt), (3) lifestyle (e.g., risk-taking, rebelliousness, impulsive), and (4) antisocial (but see Hare, 1991 for a two-factor model). To the extent that psychopathy varies continuously along these facets, we may expect that subclinical levels of variability in psychopathic traits map onto behavioral and neural processes found to be dysfunctional in criminal psychopaths. Indeed, individual differences in psychopathic traits amongst community samples have been associated with decreased amygdala reactivity to facial expressions of emotion as well as during emotional and moral decision-making tasks (Glenn, Raine, \& Schuq, 2009; Gordon, Baird, \& End, 2004; Rilling et al., 2007).

Although the above research has focused on deficits in emotional behaviors and altered amygdala reactivity, recent work examining reward processing has linked ventral striatum (VS) function with psychopathy and broader psychopathic traits (Buckholtz et al., 2010). The VS, encompassing the nucleus accumbens and ventromedial regions of the caudate and putamen, is involved in processing the motivational salience of stimuli, shaping reward expectancy and prediction, and modulating appetitive behaviors (Berridge \& Robinson, 2003). A recent study reported that individual differences within a community sample in impulsive-antisocial psychopathic traits were positively correlated with VS reactivity during anticipation of monetary reward (Buckholtz et al., 2010). Thus, individual differences in psychopathic traits may reflect altered reactivity of not only the amygdala to threat but also the VS to reward.

Despite progress in identifying the neural substrates of criminal psychopathy and variation of psychopathic traits in the general population (Koenigs, Baskin-Sommers, Zeier, \& Newman, 2011), there are a number of outstanding issues. First, and most notably, the very small sample sizes in previous neuroimaging studies limit the extent to which their findings may generalize to the broader variability exhibited across facets of psychopathy in the general population. The small sample sizes also preclude examination of gender-specific relationships, which is relevant given the male bias in psychopathy (Cale \& Lilienfeld, 2002). Indeed, some work indicates that psychopathic traits are linked to an attenuated neuroendocrine response to a psychosocial stressor, but only among men (O'Leary, Loney, \& Eckel, 2007). Similarly, Isen et al. (2010) reported blunted skin conductance responses during a passive auditory task among boys (but not girls) scoring relatively high on a self-report measure of psychopathic traits. Collectively, these findings raise the possibility of gender-specific associations between psychopathic traits and threat- and reward-related neural function.

Another clear limitation is that many of the existing studies have focused on participants who express phenotypic extremes of psychopathy (e.g., incarcerated criminal psychopaths or individuals in the top $10 \%$ of callous-unemotional traits) further limiting generalizability to broader variability in psychopathic traits. Third, while separate lines of evidence indicate that psychopathic traits are linked to dysfunctional threatand reward-related brain function, it is unclear to what extent each of these processes contributes to a unique proportion of variability in psychopathic traits in the same sample. Finally, despite evidence for the multidimensionality of psychopathy (Hare \& Neumann, 2005, 2008), few studies have examined how variability in each of the facets of psychopathy maps onto individual differences in brain function.

In the current study, we address the above outstanding issues in a sample of 200 young adults by assessing relationships between self-reported psychopathic traits and threat- and reward-related brain function measured with blood oxygen level-dependent (BOLD) functional magnetic resonance imaging (fMRI). In accordance with previous evidence in volunteers (Gordon et al., 2004), we predicted that the interpersonal and affective facets of psychopathy would be associated with decreased amygdala reactivity to fearful facial 
expressions. In addition, given that the lifestyle facet of psychopathy has been linked to reactive aggression (Hall, Benning, \& Patrick, 2004) and that reactively aggressive individuals have been shown to demonstrate amygdala hyperreactivity to angry facial expressions (Coccaro, McCloskey, Fitzgerald, \& Phan, 2007) we hypothesized that this facet would be positively correlated with amygdala reactivity to angry facial expressions. Consistent with recent evidence (Buckholtz et al., 2010), we further predicted that the lifestyle and antisocial facets of psychopathy would be positively correlated with reward-related VS reactivity. Finally, given previous gender-specific patterns of findings (O'Leary et al., 2007; Isen et al., 2010), we predicted that associations between psychopathic traits and brain function would be more robust in men.

\section{MATERIALS AND METHODS}

\section{Participants}

A total of 200 subjects (117 women; mean age $19.65 \pm 1.30$ years) completed an ongoing parent protocol, the Duke Neurogenetics Study (DNS), which assesses a wide range of behavioral and biological traits among non-patient, young adult, student volunteers. Participants qualified for the DNS if they were free of the following study exclusions: (1) medical diagnoses of cancer, stroke, diabetes requiring insulin treatment, chronic kidney or liver disease, or lifetime history of psychotic symptoms; (2) use of psychotropic, glucocorticoid, or hypolipidemic medication; and (3) conditions affecting cerebral blood flow and metabolism (e.g., hypertension). Diagnosis of any current Diagnostic and Statistical Manual of Mental Disorders (DSM-IV) Axis I disorder (First, Spitzer, Gibbon, \& Williams, 1996) was not an exclusion criterion as the DNS seeks to establish broad variability in multiple behavioral phenotypes related to psychopathology (e.g., impulsivity, aggression, anxiety). All participants provided informed consent in accord with Duke University guidelines and were in good general health and free of study exclusions.

\section{Self-Report Psychopathy-Short Form (SRP-SF)}

The Self-Report Psychopathy-Short Form (SRP-SF) is a 29-item questionnaire assessing psychopathic traits (Paulhus, Neumann, \& Hare, in press). Participants are asked to respond on the extent to which they agree with each statement using a 5-point Likert scale $(1=$ disagree strongly, $5=$ agree strongly $)$. To provide a test of the four-factor Self-Report Psychopathy (SRP) model of psychopathy, the SRP items were set to load on their respective factors: Interpersonal (items 7,9,10,15,19,23,26), Affective $(3,8,13,16,18,24,28)$, Lifestyle $(1,4,11,14,17,21,27)$, and Antisocial $(2,5,6,12,22,25,29)$. Given the ordinal nature of the items, robust weighted least-squares estimation was used. Model fit results indicated good fit, $\chi^{2}(344)=631.63$, comparative fit index $(\mathrm{CFI})=.94$, root mean square error of approximation $($ RMSEA $)=.06$, consistent with previous SRP research (Paulhus et al., in press) (note that the standardized root mean square residual (SRMR) is not available for models with ordered categorical data). The interpersonal facet consists of items such as "I have pretended to be someone else in order to get something," "I would get a kick out of scamming someone," and "Sometimes you have to pretend you like people to get something out of them." The affective facet consists of items such as "People sometimes say that I'm cold-hearted," "I never feel guilty over hurting others," and "I sometimes dump friends that I don't need anymore." The lifestyle facet includes items such as "I'm a rebellious person," "I've often done something dangerous just for the thrill of it," and "I admit that I often mouth off without thinking." The antisocial facet includes items such as "I have broken into a building or vehicle in order to steal something or vandalize," "Every now and then I carry a weapon (knife or gun) for protection," and "I was convicted of a serious crime." Reliability was acceptable for all four facets (Cronbach's alpha $>.74$; mean inter-item correlation $>.30$ ). Sum scores from each facet were used in subsequent analyses.

In addition to being strongly correlated with the Psychopathy Checklist-Revised (PCL-R) (Paulhus et al., in press), the gold standard clinical interview for psychopathy, the SRP recently has been shown to be highly correlated with the Youth Psychopathic Inventory, an established psychopathy self-report, as well as with number of criminal offenses and measures of externalizing psychopathology (Neumann \& Pardini, in press) in a large community sample of delinquent young adults. In addition, in two large general population samples from the United Kingdom and Australia, respectively, the SRP has been shown to be significantly associated with disturbances in moral reasoning and empathy (Seara-Cardoso, Neumann, Roiser, McCrory, \& Viding, 2012), as well as alcohol use and aggression (Watt \& Brooks, 2011). 
Overall, the strong modeling results for the SRP in the current sample are consistent with other published studies on the SRP (Mahmut, Menictas, Stevenson, \& Homewood, 2011; Neumann \& Pardini, in press; Seara-Cardoso et al., 2012; Williams, Paulhus, \& Hare, 2007) attesting to its clear and reliable latent factor structure, which is notably lacking for other selfreport measures such as the Psychopathic Personality Inventory (Neumann, Uzieblo, Grombez, \& Hare, in press).

\section{Amygdala reactivity paradigm}

Our fMRI challenge paradigm has been used extensively to elicit a robust and replicable amygdala response across an array of experimental protocols and sample populations (e.g., Fisher et al., 2006, 2009; Hariri et al., 2002, 2005; Manuck, Brown, Forbes, \& Hariri, 2007; Zhou et al., 2008). The experimental fMRI paradigm consisted of four blocks of a perceptual face-matching task interleaved with five blocks of a sensorimotor control task. The DNS version of this paradigm consists of one block each of fearful, angry, surprised, and neutral facial expressions presented in a pseudorandom order across participants. During face-matching blocks, participants view a trio of faces and select one of two faces (on the bottom) identical to a target face (on the top). Each face processing block consists of six images, balanced for gender, all of which were derived from a standard set of pictures of facial affect (Ekman \& Friesen, 1976). During the sensorimotor control blocks, participants view a trio of simple geometric shapes (circles and vertical and horizontal ellipses) and select one of two shapes (bottom) that are identical to a target shape (top). Each sensorimotor control block consists of six different shape trios. All blocks are preceded by a brief instruction ("Match Faces" or "Match Shapes") that lasts $2 \mathrm{~s}$. In the task blocks, each of the six face trios is presented for $4 \mathrm{~s}$ with a variable interstimulus interval (ISI) of 2-6 s (mean = $4 \mathrm{~s}$ ) for a total block length of $48 \mathrm{~s}$. A variable ISI is used to minimize expectancy effects and resulting habituation, and maximize amygdala reactivity throughout the paradigm. In the control blocks, each of the six shape trios is presented for $4 \mathrm{~s}$ with a fixed ISI of $2 \mathrm{~s}$ for a total block length of $36 \mathrm{~s}$. Total task time is $390 \mathrm{~s}$. In the current study, we focused our analyses on fear and anger blocks. We restricted our analyses to fearful and angry facial expressions because they represent honest indicators of an ecologically valid threat and in this context we interpret the amygdala activation elicited by our task as being threat related.

\section{VS reactivity paradigm}

Our blocked design paradigm consisted of pseudorandom presentation of trials wherein participants played a card guessing game and received positive or negative (i.e., correct or incorrect guess) feedback for each trial (Hariri et al., 2009). During each trial, participants have $3 \mathrm{~s}$ to guess, via button press, whether the value of a visually presented card is higher or lower than 5 (index and middle fingers, respectively). The numerical value of the card is then presented for $500 \mathrm{~ms}$ and followed by the appropriate feedback (green upward-facing arrow for positive feedback; red downward-facing arrow for negative feedback) for an additional $500 \mathrm{~ms}$. A crosshair is then presented for $3 \mathrm{~s}$ for a total trial length of $7 \mathrm{~s}$. Each block is composed of five trials, and the paradigm consists of three blocks each of predominantly positive feedback (80\% correct) and three of predominantly negative feedback (20\% correct) interleaved with three control blocks. During control blocks, participants are instructed to simply make button presses during the presentation of an " $\mathrm{x}$ " (3 s), which is followed by an asterisk (500 ms) and a yellow circle $(500 \mathrm{~ms})$. Each block is preceded by an instruction of "Guess Number" (positive or negative feedback blocks) or "Press Button" (control blocks) for $2 \mathrm{~s}$ resulting in a total block length of $38 \mathrm{~s}$ and a total task length of $342 \mathrm{~s}$

Participants are told that their performance on the card game will determine a monetary reward to be received at the end of the game and are unaware of the fixed outcome probabilities associated with task blocks. We include one incongruent trial within each task block (e.g., one of five trials during positive feedback blocks is incorrect, resulting in a negative feedback) to prevent participants from anticipating the feedback for each trial and to maintain participants' engagement and motivation to perform well.

\section{BOLD fMRI data acquisition}

Each participant was scanned using a researchdedicated General Electric MR750 3T scanner at the Duke-UNC Brain Imaging and Analysis Center. This scanner is equipped with high-power, highduty cycle $50 \mathrm{mT} / \mathrm{m}$ gradients at $200 \mathrm{~T} / \mathrm{m} / \mathrm{s}$ slew rate, and an eight-channel head coil for parallel imaging at high bandwidth up to $1 \mathrm{MHz}$. A semiautomated high-order shimming program was used to ensure global field homogeneity. A series of 34 interleaved axial functional slices aligned with the anterior commissure-posterior commissure (AC-PC) 
plane were acquired for full-brain coverage using an inverse-spiral pulse sequence to reduce susceptibility artifact (TR/TE/flip angle $=2000 \mathrm{~ms} / 30 \mathrm{~ms} / 60$; FOV $=240 \mathrm{~mm} ; 3.75 \times 3.75 \times 4$ mm voxels; interslice skip $=0$ ). Four initial RF excitations were performed (and discarded) to achieve steady-state equilibrium. To allow for spatial registration of each participant's data to a standard coordinate system, highresolution three-dimensional structural images were acquired in 34 axial slices coplanar with the functional scans $(\mathrm{TR} / \mathrm{TE} /$ flip angle $=7.7 \mathrm{~s} / 3.0 \mathrm{~ms} / 12$; voxel size $=0.9 \times 0.9 \times 4 \mathrm{~mm} ; \mathrm{FOV}=240 \mathrm{~mm}$, interslice skip $=0)$.

\section{BOLD fMRI data preprocessing}

Preprocessing was conducted using SPM8 (www.fil. ion.ucl.ac.uk/spm). Images for each participant were realigned to the first volume in the time series to correct for head motion, spatially normalized into a standard stereotactic space (Montreal Neurological Institute template) using a 12-parameter affine model (final resolution of functional images $=2 \mathrm{~mm}$ isotropic voxels), and smoothed to minimize noise and residual differences in gyral anatomy with a Gaussian filter set at $6 \mathrm{~mm}$ full width at half maximum. Voxel-wise signal intensities were ratio normalized to the whole-brain global mean.

Because of the relatively extensive signal loss and noise typically observed in amygdala and VS, single-subject BOLD fMRI data were included in subsequent analyses only if there was a minimum of $90 \%$ signal coverage in the amygdala bilaterally and in the VS bilaterally (see Nikolova, Bogdan, Brigidi, \& Hariri, 2012). These criteria, which were selected to maximize signal in our Regions of Interest (ROI) while also maintaining sufficient sample sizes to test our hypotheses, resulted in 199 single-subject data sets (114 women) with adequate coverage in the amygdala and 171 singlesubject data sets (103 women) with adequate coverage in the VS.

Variability in single-subject whole-brain functional volumes was determined using the Artifact Recognition Toolbox (http://www.nitrc.org/projects/ artifact_detect). Individual whole-brain BOLD fMRI volumes meeting at least one of two criteria were assigned a lower weight in the determination of taskspecific effects: (1) significant mean volume signal intensity variation (i.e., within-volume mean signal greater or less than $4 \mathrm{SD}$ of mean signal of all volumes in time series) and (2) individual volumes where scan-to-scan movement exceeded $2 \mathrm{~mm}$ translation or $2^{\circ}$ rotation in any direction.
After preprocessing, linear contrasts using canonical hemodynamic response functions were used to estimate condition-specific (i.e., fear $>$ shapes, angry $>$ shapes, and positive $>$ negative feedback) BOLD responses for each individual and paradigm. These individual contrast images (i.e., weighted sum of the beta images) were then used in second-level randomeffects models to determine mean condition-specific neural reactivity using one-sample $t$-tests with a voxellevel statistical threshold of $p<.05$, familywise error (FWE) corrected for multiple comparisons across the entire search volume. Contrast estimates were then extracted from functional clusters exhibiting a main effect of task using the above threshold within anatomically defined amygdala and VS regions of interest. The VS ROI was constructed using the Talairach Daemon option of the WFU PickAtlas Tool v2.4. Two spheres of $10 \mathrm{~mm}$ radius were created around Montreal Neurological Institute (MNI) coordinates $x= \pm 12$, $y=12$, and $z=-10$ to encompass the VS in the right and left hemispheres, respectively. Because of the structural and functional heterogeneity of the amygdala (Davis \& Whalen, 2001), we examined the ventral and dorsal amygdala independently to determine whether individual differences in psychopathy facets map onto the amygdala's principal input and output regions, respectively. This approach is justified based on previous imaging research indicating that individual difference factors map onto specific regions of the amygdala (Carré, Fisher, Manuck, \& Hariri, in press; Etkin et al., 2004; Hyde, Gorka, Manuck, \& Hariri, 2011; Manuck et al., 2010).

We constructed hemisphere-specific ROIs using Marsbar (v 0.41) for the ventral amygdala, which encompass the basolateral complex, and for the dorsal amygdala, which encompass the central nucleus as well as the sublenticular extended amygdala and nucleus basalis of Meynert. The ventral amygdala ROIs were anchored by the MNI coordinates $x= \pm 21$, $y=-3$, and $z=-23$, with widths of $14 \mathrm{~mm}, 6 \mathrm{~mm}$, and $6 \mathrm{~mm}$ along the $x$-, $y$-, and $z$-axes, respectively. The total volume of the ventral amygdala ROI was $1024 \mathrm{~mm}^{3}$ in each hemisphere. The dorsal amygdala ROIs were anchored by the MNI coordinates $x= \pm 21$, $y=-4$, and $z=-13$, with widths of $14 \mathrm{~mm}, 8 \mathrm{~mm}$, and $10 \mathrm{~mm}$ along the $x$-, $y$-, and $z$-axes, respectively. The total volume of the dorsal amygdala ROI was $1920 \mathrm{~mm}^{3}$ in each hemisphere. The reported widths reflect the total for the ROI along each axis and are centered on the MNI coordinate anchoring each axis (i.e., with $x=21$ and width $=14 \mathrm{~mm}$, the range of coordinates included along that axis of the ROI are from $x=14$ to $x=28$ ). The posterior extent of both the dorsal and ventral amygdala was carefully defined to exclude the hippocampus. 


\section{Statistical analyses}

BOLD parameter estimates exhibiting a main effect of task (e.g., fear $>$ shapes, anger $>$ shapes, positive $>$ negative feedback) were extracted from the ROIs using the volume of interest (VOI) tool in SPM8. For all ROIs, BOLD parameter estimates were extracted from the peak activation voxel within the left and right hemispheres. In addition to producing the necessary values for our path models, extracting parameter estimates from functional clusters activated by our fMRI paradigms rather than clusters specifically correlated with our independent variables of interest precludes the possibility of any correlation coefficient inflation that may result from capitalizing on the same data twice (Viviani, 2010). We have successfully used this more conservative and rigorous analytic strategy in recent studies (Carré et al., in press; Hyde et al., 2011).

Path modeling was used to examine the extent to which psychopathic traits mapped onto variation in amygdala and VS reactivity. This approach enabled us to control for the overlap of each facet of psychopathy while also simultaneously examining their unique associations with brain function (e.g., amygdala reactivity to fearful faces, amygdala reactivity to angry faces, VS reactivity to positive feedback). By estimating one model simultaneously, we reduce the chances of finding associations by chance via many zeroorder correlations. Thus, the advantage of path analysis within Structural Equation Modeling software is that we are able to test all outcomes simultaneously (akin to multivariate analysis of variance (MANOVA)), as well as evaluate the fit of our model (which was acceptable). Path modeling in which there are multiple outcomes has a built in way (i.e., model fit) of penalizing for testing many paths that are not significant. In addition, the main focus of our study is to uncover psychobiological processes through model testing rather than simple null hypothesis testing. In this case, the approach is to make our model best account for the data (make a model that fits well by creating small residuals). Again, our acceptable fit reflects a good fit of the model to the data and is evidence that our findings are replicable (in the null hypothesis significance testing vein), as well as fit the data well.

The path model is presented in Figure 3 and was estimated using Mplus 6.0 (Muthen \& Muthen, 2010) utilizing maximum-likelihood estimation, which can accommodate the small amount of missing data (covariance coverage $>.85$ for all variables, $N=$ 171-199). We excluded a total of 24 extracted ROI values (fear $>$ shapes, one right dorsal amygdala, two left dorsal amygdala, two right ventral amygdala, two left ventral amygdala; anger $>$ shapes, two right dorsal amygdala, four left dorsal amygdala, one right ventral amygdala, three left ventral amygdala; positive $>$ negative feedback, three right VS, four left VS) and treated them as "missing" in the model because they were identified as statistical outliers (i.e., \pm 3 SD from the mean). In this model, gender was used as a covariate and all of the covariances between SRP facets were modeled, as well as the covariance between some of the ROI values. All paths possible between each psychopathy facet and each ROI measure were modeled and retained in the final model (i.e., we tested whether the interpersonal facet predicted right and left dorsal and ventral amygdala reactivity to angry faces, right and left dorsal and ventral amygdala reactivity to fearful faces, and right and left VS reactivity to positive feedback). We specified the model with psychopathy facets as predictors rather than outcomes to statistically control for the overlap of each facet, which enabled us to examine each facet's unique association with each ROI. Finally, we examined possible gender moderation of findings through multi-group modeling. A series of model analyses were run in which parameters were first constrained to be equal across groups and then model fit results were compared to the same model when parameters were not constrained to be equal. We tested for an overall gender effect (all predictor paths constrained vs. all free) and did not find an omnibus gender moderation effect $\left(\chi^{2}=19.77, \mathrm{df}=24, \mathrm{~ns}\right)$.

Although our primary interest was in testing the significance of each path in the model, path modeling generated in Mplus (Muthen \& Muthen, 2010) also provides statistical tests of the fit of the hypothesized model to the observed data, which fit within a multivariate modeling goal of maximizing fit of a hypothesized model to the data. In our analyses, fit of a path model was considered acceptable if it had a RMSEA smaller than .08 (less than .05 being ideal), a CFI of greater than .90 , and a SRMR close to zero. A nonsignificant $\chi^{2}$-fit statistic is also considered ideal.

\section{RESULTS}

\section{Amygdala reactivity}

Consistent with prior reports (Fisher et al., 2006, 2009; Hariri et al., 2002, 2005; Manuck et al., 2007; Zhou et al., 2008), perceptual processing of both fearful and angry facial expressions elicited robust bilateral dorsal and ventral amygdala reactivity (see Figure 1a-d). There was no significant differential amygdala reactivity to fearful or angry facial expressions at the group level. 
(a)

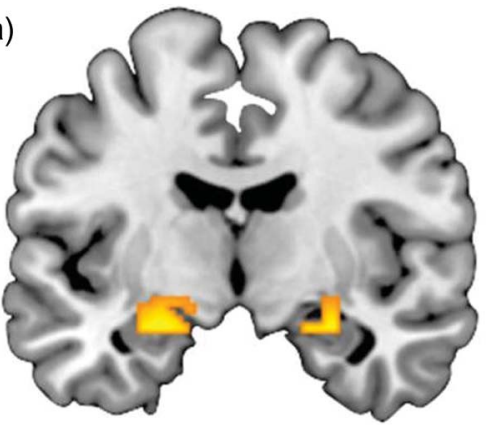

(c)

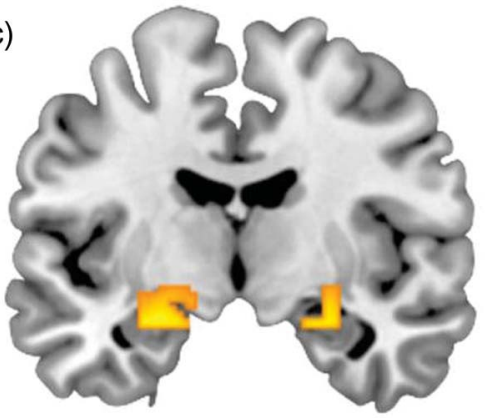

(b)

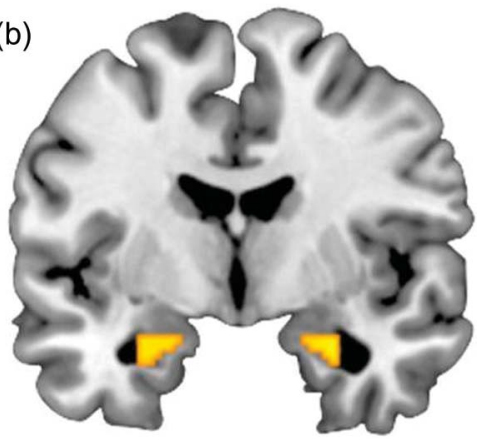

(d)

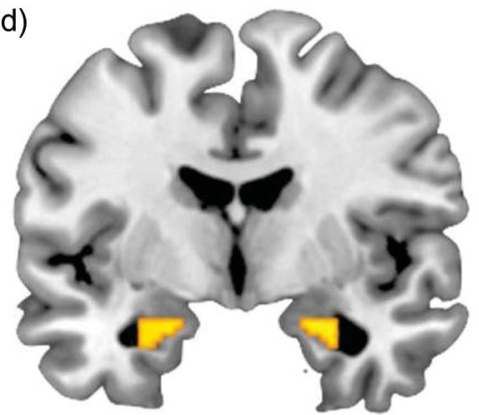

Figure 1. Coronal overlays on structural images illustrating threat-related neural reactivity. (a) Bilateral dorsal amygdala reactivity to fearful faces > shapes: right hemisphere maximal voxel MNI coordinates $x=28, y=-8$, and $z=-18 ; 77$ voxels; $t=12.13, p<.05$, FWE corrected; left hemisphere maximal voxel MNI coordinates $x=-24, y=-8$, and $z=-18$; 121 voxels; $t=11.55, p<.05$, FWE corrected. (b) Bilateral ventral amygdala reactivity to fearful faces > shapes: right hemisphere maximal voxel MNI coordinates $x=28, y=-6$, and $z=-22 ; 43$ voxels; $t=11.54, p<.05$, FWE corrected; left hemisphere maximal voxel MNI coordinates $x=-26, y=-6$, and $z=-20 ; 56$ voxels; $t=10.91$, $p<.05$, FWE corrected. (c) Bilateral dorsal amygdala reactivity to angry faces $>$ shapes: right hemisphere maximal voxel MNI coordinates $x=22, y=-6$, and $z=-18 ; 100$ voxels; $t=12.88, p<.05$, FWE corrected; left hemisphere maximal voxel MNI coordinates $x=-24$, $y=-8$, and $z=-18 ; 157$ voxels; $t=11.88, p<.05$, FWE corrected. (d) Bilateral ventral amygdala reactivity to angry faces $>$ shapes: right hemisphere maximal voxel MNI coordinates $x=28, y=-6$, and $z=-22$; 43 voxels; $t=12.70, p<.05$, FWE corrected; left hemisphere maximal voxel MNI coordinates $x=-20, y=-6$, and $z=-20 ; 56$ voxels; $t=11.48, p<.05$, FWE corrected. Note: Dorsal and ventral amygdala are displayed at $y=-8$ and $y=-6$, respectively.

\section{VS reactivity}

Also consistent with prior studies (Forbes et al., 2009; Hariri et al., 2006, 2009), differential positive relative to negative feedback in response to a simple card guessing game elicited robust bilateral VS reactivity (see Figure 2).

\section{Associations between self-reported psychopathy facets and brain reactivity}

No bivariate associations were observed between the four facets of psychopathy identified using the SRP questionnaire and amygdala reactivity to either angry or fearful faces (all $p \mathrm{~s}>.10$ ) or VS reactivity (all $p \mathrm{~s}>.06$ ). The results from the path model are presented in Figure 3. Note that results in this figure focus on the dorsal amygdala as findings were more robust in this region. Moreover to avoid problems with multicollinearity and multiple comparisons, we focused our analyses on the dorsal amygdala. However, we obtained similar results when including dorsal and ventral amygdala (Supplemental Figure 1 is available via the 'Supplementary' tab on the article's online page (http://dx.doi.org/10.1080/17470919. 2012.703623).). The main model (with dorsal amygdala values) fits the data very well $\left(\chi^{2}=8.721\right.$, $\mathrm{df}=8, p>.05$; CFI $=.998$; RMSEA $=.021$; SRMR $=.016$ ), even though nonsignificant paths had not been trimmed. The path from gender to right dorsal amygdala reactivity to fearful faces was statistically significant indicating that men demonstrated increased reactivity to this contrast compared with women. In addition, the model indicates that when accounting for the overlap of the four facets of psychopathy, the interpersonal facet was negatively associated with right dorsal amygdala reactivity to fearful faces, and the lifestyle facet was positively associated with right dorsal amygdala reactivity to angry faces but negatively associated with left VS reactivity to positive feedback. The 


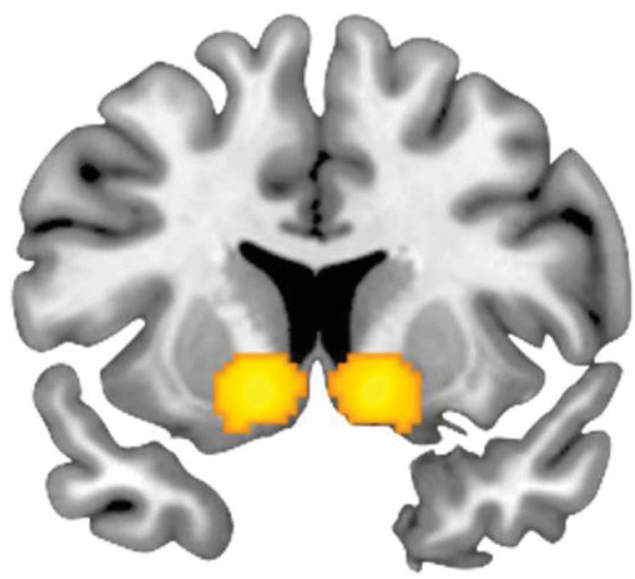

Figure 2. Coronal overlay on structural image illustrating rewardrelated VS reactivity. Bilateral VS reactivity to positive $>$ negative feedback: right hemisphere maximal voxel MNI coordinates $x=12$, $y=10$, and $z=-8 ; 376$ voxels; $t=7.30, p<.05$, FWE corrected; left hemisphere maximal voxel MNI coordinates $x=-12, y=10$, and $z=10 ; 343$ voxels; $t=6.18, p<.05$, FWE corrected. Displayed at $y=10$.

antisocial facet was positively associated with left VS reactivity. ${ }^{1}$

\section{Gender-specific associations}

Given the focus on men in most psychopathy research, we examined possible gender differences in the relationships between brain function and psychopathy facets. To examine possible gender differences in our path model, we examined this model using a multigroup path model in which the model is estimated

\footnotetext{
1 Predictor variables are not required to be normally distributed in multiple regression and path analyses, and thus, our findings should not be influenced by non-normality of SRP facets. Nevertheless, we decided to run additional path analyses using procedures that are robust to violations of normality and provide unbiased SEs regardless of distributional properties. First, using multiple linear regression (MLR) estimation in Mplus 6.0 (maximum likelihood with robust SEs), results indicated that every path that was significant in our original analyses continued to be significant, except that the association between the antisocial facet and left VS reactivity became nonsignificant. In addition, using bootstrapping for the SEs, which makes no assumptions about the distribution of the data, every path continues to be significant except the association between the antisocial facet and left VS reactivity is nonsignificant and the negative association between the lifestyle facet and left VS reactivity became $p=.05$ instead of $p<.05$. Finally, if the skew in the distributions were a problem, one would expect to see evidence of heteroscedasticity when examining the residuals. However, examination of the residual plots revealed no evidence of heteroscedasticity. In summary, given the convergence of findings across the several different analytic procedures, we are very confident that the distribution of our variables has not undermined the reliability of our findings.
}

for both groups and interaction effects are tested by examining whether individual paths differ significantly between each group through $\chi^{2}$ difference tests. An identical model shown in Figure 3 (but without gender as a covariate) was run simultaneously for men $(N=82)$ and women $(N=117)$ in which all paths were allowed to vary between groups. Model fit was similar to the non-grouped model $\left(\chi^{2}=19.379\right.$, $\mathrm{df}=16, p>.05 ; \mathrm{CFI}=.991$, RMSEA $=.046$, SRMR $=.033)$. In this model, men demonstrated the following significant relationships between variables: the interpersonal facet was negatively associated (trend level) with right dorsal amygdala reactivity to fear $(B=-.013, \mathrm{SE}=.007, p=.08)$ and the lifestyle facet was positively associated with right dorsal amygdala reactivity to anger $(B=.041, \mathrm{SE}=.015$, $p<.01)$. In women, the following significant relationships were evident: the affective facet was positively associated with right VS reactivity $(B=.014$, $\mathrm{SE}=.006, p=.015)$ and there was a trend toward a negative association between the interpersonal dimension and the right dorsal amygdala reactivity to fear faces $(B=-.018, \mathrm{SE}=.009, p=.05)$ and the lifestyle facet was negatively associated (trend level) with right VS reactivity to reward $(B=-.009$, $\mathrm{SE}=.005, p=.06)$ and with left VS reactivity to reward $(B=-.010, \mathrm{SE}=.005, p=.03)$.

However, when paths were compared using $\chi^{2}$ difference tests, there was no omnibus gender moderation effect and only two paths differed significantly across groups. When all predictor "on" statements were free versus fixed the model fit did not improve $\left(\chi^{2}\right.$ difference $\left.=19.768, \mathrm{df}=24, p>.05\right)$; the fixed path model actually fits better meaning that the overall model did not fit better when allowed to vary across groups. For the most part each path was also equivalent across genders when the path was individually fixed and freed and compared via $\chi^{2}$ difference tests. However, two paths did demonstrate evidence of significant moderation by gender. The path between the lifestyle facet and right dorsal amygdala reactivity to anger was only significant in men and not women $\left(\chi^{2}\right.$ difference $\left.=4.9, \mathrm{df}=1, p<.05\right)$. The trend between the affective facet and right VS reactivity was present in women but not in men $\left(\chi^{2}\right.$ difference $=5.15, \mathrm{df}=1, p<.05)$. Thus these two paths demonstrate significant moderation by gender.

\section{Controlling for impulsivity}

To examine the possible confound that impulsivity may have on our findings, we examined a path model in which impulsivity (as measured by Barrett's 


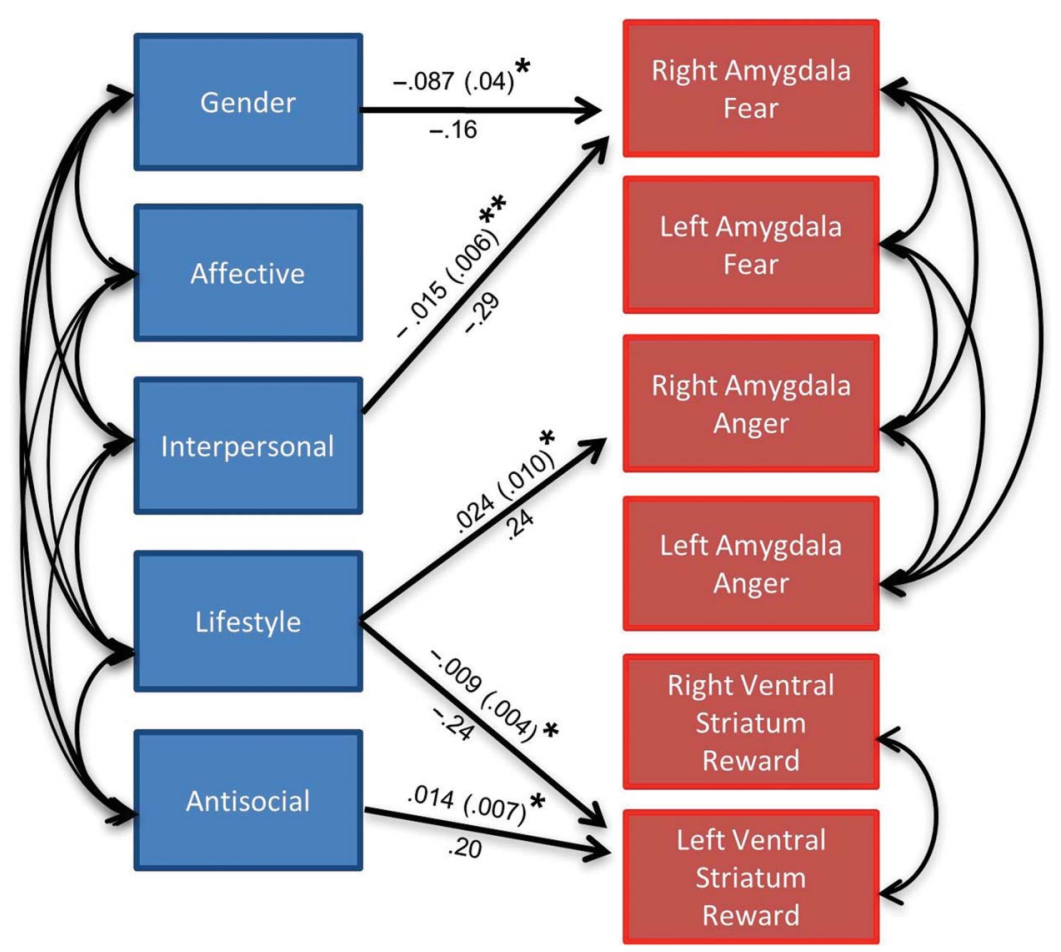

Figure 3. Self-reported dimensions of psychopathy are associated with threat- and reward-related neural function. A path model tested the relationship between dimensions of psychopathy as measured by the SRP-SF and amygdala reactivity to fear faces versus shapes, anger faces versus shapes, and VS response to positive versus negative feedback during a reward task. Note that in all paths from each SRP-SF facet and each neural variable were modeled but for clarity, only significant or trend level paths are displayed in this figure. Gender was used as a covariate. Note also that covariance between amygdala reactivity variables and VS variables were initially modeled, but as these covariances were nonsignificant, they were trimmed from the final model. The model included $N=199$ individuals. The fit for this model was excellent: $\left(\chi^{2}=8.721, \mathrm{df}=8, p>.05 ; \mathrm{CFI}=.998 ; \mathrm{RMSEA}=.021 ; \mathrm{SRMR}=.016\right)$. Note: This model represents only paths significant at $p<.05$. There was one path that was significant at trend level (affective facet predicting right VS reactivity to reward, $B=.008, \mathrm{SE}=.005, p=.09$ ). Numbers above lines in the model are unstandardized parameter estimates with SEs in parentheses. Numbers below lines are standardized parameter estimates. ${ }^{* *} p<.01,{ }^{*} p<.05$.

Impulsiveness Scale (Patton, Stanford, \& Barratt, 1995)) was controlled for as a predictor (similar to how gender was modeled in the original path model). This model was consistent with the model presented in the main results, except that the relationship between the antisocial facet and left VS reactivity became a trend and the lifestyle facet became associated (at a trend level) with right VS reactivity. This model also fits the data well $\left(\chi^{2}=10.464, \mathrm{df}=8, p>.05, \mathrm{CFI}=.994\right.$, RMSEA $=.040$, SRMR $=.040)$.

\section{DISCUSSION}

Our findings are largely consistent with theoretical models and empirical evidence demonstrating associations between threat-related amygdala reactivity, reward-related VS reactivity, and individual differences in psychopathic traits (Blair, 2007; Buckholtz et al., 2010; Glenn et al., 2009; Gordon et al., 2004).
More importantly, our data offer several novel contributions. First, our large sample of men and women allowed us to examine relationships between variability in psychopathic traits and test for gender differences in any emerging patterns. Notably, previous neuroimaging studies in the psychopathy literature have been conducted on relatively small sample sizes (Koenigs et al., 2011), and thus, our study represents the first large-scale examination of neural correlates of psychopathic traits. Second, our focus on a cohort of healthy young adult volunteers allowed for an examination of broader phenotypic variability in psychopathic traits. Third, the application of two well-characterized BOLD fMRI paradigms allowed us to simultaneously examine associations between psychopathy and both threat- and reward-related brain function. Finally, by utilizing a multidimensional measure of psychopathy, we were able to examine the unique and shared associations between brain function and each of four facets of psychopathy. 
The unique negative correlation between amygdala reactivity to fearful facial expressions and the interpersonal facet is generally consistent with prior associations of psychopathic traits with reduced amygdala reactivity to the distress of others (Blair, 2007; Gordon et al., 2004; Jones et al., 2009; Marsh et al., 2008). This negative association may provide a better understanding of how the elements of this facet (e.g., conning, deceptive) can be accounted for in terms of brain function. Decreased amygdala processing of distress cues may underlie the persistent instrumental aggression displayed by psychopathic individuals. Notably, when all four facets of psychopathy are considered, the interpersonal facet is the only unique predictor of individual differences in instrumental aggression (Laurell, Belfrage, \& Hellstrom, 2010; Vitacco, Neumann, Caldwell, Leistico, \& Van Rybroek, 2006). Blair (1995) has proposed the violence inhibition mechanism (VIM) to account for both the blunted amygdala response to distress cues and the instrumental aggression displayed by psychopaths. According to the VIM, moral socialization occurs through the pairing of distress cues (unconditioned stimuli, US; e.g., sad or fearful faces) with representations of the acts leading to the distress (conditioned stimuli, CS; e.g., hitting another person). The inability to learn such CS-US pairings, a critical function of the amygdala, may contribute to a dysfunctional VIM and heightened instrumental aggression. However, the extent to which amygdala function mediates the association between impaired CS-US learning and instrumental aggression is currently unclear. Interestingly, as past studies have not examined the interpersonal and affective factors separately, our study is the first to suggest that the interpersonal rather than the affective component may be driving this association.

In contrast to the above, there was a significant positive association between amygdala reactivity to angry facial expressions and the lifestyle facet of psychopathy. This finding indicates that psychopathic traits may not be exclusively associated with amygdala hyporeactivity, and that it is critical to consider both the nature of the threat-related stimuli (e.g., distress-fearful expressions versus interpersonal challenge-angry expressions) and the specific facet of psychopathic traits. The lifestyle facet of psychopathy has been specifically linked to reactive aggression among forensic and non-forensic samples (Hall et al., 2004). In addition, reactively aggressive individuals demonstrate heightened amygdala reactivity to angry faces (Coccaro et al., 2007). The divergent correlations observed between the interpersonal facet and amygdala reactivity to fearful facial expressions and the lifestyle facet and amygdala reactivity to angry facial expressions are consistent with the hypothesis that relatively blunted amygdala reactivity to distress may be a risk factor for instrumental aggression, whereas heightened amygdala reactivity to interpersonal challenge may be a risk factor for reactive aggression (Carré et al., in press). Future work in which individual differences in psychopathic traits are assessed along with laboratory measures of instrumental and reactive aggression (e.g., Carré, Gilchrist, Morrissey, \& McCormick, 2010; Nouvion, Cherek, Lane, Tcheremissini, \& Lieving, 2007) during fMRI are needed to test such mediation models.

Because of the structural and functional heterogeneity of the amygdala (Davis \& Whalen, 2001), we constructed ROIs encompassing the ventral and dorsal regions to examine whether the amygdala's principal input and output regions mapped onto variation in psychopathic traits. Our path models indicated that the interpersonal facet of psychopathy was negatively correlated with both ventral and dorsal amygdala reactivity to fearful faces (see Figure 1 and Supplementary Figure 1). Thus, although subregions of the amygdala serve different functions in fear processing (e.g., Tye et al., 2011), our findings suggest a general hyporesponsiveness of the amygdala during fear processing among individuals scoring high on the interpersonal facet. In addition, our results suggested that the lifestyle facet of psychopathy was positively correlated with dorsal amygdala reactivity (but not ventral amygdala reactivity) to angry faces. The significant effect observed in the dorsal amygdala is similar to previous work finding specific associations between dorsal amygdala reactivity (but not ventral) to threat-related facial expressions and individual differences in trait anger and anxiety (Carré et al., in press; Etkin et al., 2004; Hyde et al., 2011). Nevertheless, the effect in the ventral amygdala in the current study approached statistical significance $(p=.102$; see Supplementary Figure 1), suggesting that individuals scoring high on the lifestyle facet showed a general hyperresponsiveness of the amygdala to angry facial expressions. Collectively, these findings challenge current models of psychopathy, which suggest a general amygdala hyporeactivity to facial expressions of emotion. Specifically, we demonstrate that the direction of the association between amygdala reactivity and psychopathic traits depends critically on the particular facet of psychopathy under investigation (e.g., interpersonal vs. lifestyle) and the type of emotional expression being processed (e.g., fearful vs. angry faces).

Other work indicates that individual differences in the impulsive-antisocial factor of psychopathy are positively correlated with VS reactivity to the anticipation 
(but not the outcome) of monetary reward (Buckholtz et al., 2010). Moreover, the correlation between reward-related VS reactivity and the antisocial facet of psychopathy is independent of self-reported impulsivity (assessed via the Barratt Impulsiveness Scale). This suggests that reward-related VS reactivity may be specifically driven by the antisocial dimension of psychopathy (Buckholtz et al., 2010). In contrast, we found that after controlling for variation in general impulsivity, there was no association between the antisocial facet and VS reactivity to reward. However, we did observe a negative correlation between VS reactivity and the lifestyle facet of psychopathy (even after controlling for impulsivity). The finding of a negative correlation between VS reactivity and the lifestyle facet is particularly surprising given that previous work indicates that impulsive traits are positively correlated with VS reactivity during reward processing (Forbes et al., 2009; Hariri et al., 2006). However, closer inspection of the individual items used to derive the lifestyle facet in the current study suggests that this facet may be capturing more general risk-taking or sensation-seeking traits, rather than impulsivity per se (e.g., "I'm a rebellious person," "I've often done something dangerous just for the thrill of it," and "I enjoy doing wild things"). From this perspective, our findings are consistent with the proposal that individuals with relatively decreased VS reactivity may engage in risk-taking and sensation-seeking behaviors as a means to compensate for a hypoactive" reward circuit (Reuter et al., 2005). Our findings are consistent with recent studies indicating that youth with conduct disorder and psychopathic traits demonstrate decreased dorsal striatal reactivity during initial learning of reinforcement contingencies (Finger et al., 2011), and increased dorsal striatal reactivity to punished reversal errors compared with rewarded correct responses (Finger et al., 2008). Collectively, these findings suggest that abnormalities in the ventral and dorsal striatum may play a key role in the etiology of psychopathic traits.

Although our findings are generally consistent with prior reports (Jones et al., 2009; Marsh et al., 2008), one important methodological difference between the current study and previous imaging studies is that we simultaneously regressed brain activation onto each of the four psychopathy facets, whereas previous studies have focused mainly on bivariate associations between brain activation and psychopathy facets. In the current study, more robust effects were found within the multivariate analyses. These findings are consistent with a "suppression effect" wherein the inclusion of two or more predictors in the same model increases the effect of one or more of the variables in predicting the dependent variable (Paulhus, Robins, Trzesniewski, \& Tracy, 2004). Suppression effects are theorized to reflect the fact that shared variance between predictors is irrelevant to the outcome, and that each predictor's validity to the outcome is enhanced by partitioning out their shared variance. A good example of suppressor effects in psychopathy research was reported by Hicks and Patrick (2006) who found that the association between the interpersonal/affective dimension of psychopathy and negative emotionality became more negative and the association between the impulsive/antisocial dimension and negative emotionality became more positive when both facets were included in the same model. In this case as with our current data, it appears that these two facets of psychopathy are more predictive only after accounting for their overlapping variance.

There are some limitations to our study that should be noted. First, although we attempted to capture broader variability in psychopathy, participants scored relatively low on psychopathic traits. Thus, we cannot exclude the possibility that additional associations between SRP facets and brain function may have emerged had we sampled from a more diverse group that included individuals at the high end of the distribution. Nevertheless, our findings are consistent with evidence from individuals scoring at the extreme end of the psychopathy continuum (e.g., Finger et al., 2008, 2011; Jones et al., 2009; Marsh et al., 2008), suggesting that the inclusion of extreme phenotypes may have strengthened the associations observed in the current study. Furthermore, other evidence indicates that normal variability in psychopathic traits is associated with important behavioral outcomes. Specifically, Neumann and Hare (2008) found that even though the vast majority of their sample scored very low on the Psychopathy Checklist: Screening Version (PCL:SV), variation in PCL:SV predicted behavioral outcomes such as violence, alcohol use, and IQ. Thus, even at relatively low levels, psychopathic traits are pathological. Finally, the utility of studying normative variation in psychopathic traits is highlighted by a wealth behavioral genetic, developmental, and taxonomic studies that all point to the dimensional nature of psychopathy (Guay, Ruscio, Knight, \& Hare, 2007; Loney, Taylor, Butler, \& Iacono, 2007; Viding, Frick, \& Plomin, 2007). A second limitation is that although we found some evidence for gender-specific patterns (e.g., positive association between the lifestyle facet and amygdala reactivity to angry faces was specific to men; positive association between the affective facet and VS reactivity was specific to women), we cannot exclude the possibility that a larger sample size may have revealed more robust gender-dependent 
associations (Isen et al., 2010). Thus, future work with larger samples may uncover interesting genderspecific associations between psychopathic traits and brain function. Another limitation of our study is that the blocked design of our VS reactivity paradigm does not enable us to specifically assess the extent to which psychopathy facets map onto VS function during the anticipatory and/or consummatory phases of reward processing. Thus, a direct comparison to the findings of Buckholtz et al. (2010) is not possible.

In summary, the current study systematically assessed relationships between psychopathic traits and both threat- and reward-related brain function in a large sample of young adults. Our results are consistent with recent evidence linking psychopathic traits with abnormal amygdala and VS reactivity to threat and reward, respectively. Moreover, our findings highlight the importance of considering subfacets of the larger psychopathic construct and their simultaneous modeling when attempting to map brain-behavior relationships. Simultaneous modeling of threat- and reward-related neural function is an important step toward a more comprehensive understanding of the array of neural variability associated with psychopathy. Ultimately, it may be a combination of neural vulnerabilities (e.g., amygdala hyporeactivity to fear, hyperreactivity to anger, and VS hyporeactivity to reward) that contributes to the "full-blown manifestation" of the psychopathy syndrome.

\section{REFERENCES}

Berridge, K. C., \& Robinson, T. E. (2003). Parsing reward. Trends in Neuroscience, 26, 507-513.

Birbaumer, N., Veit, M., Lotze, M., Erb, M., Hermann, C., Grodd, W., \& Floor, H. (2005). Deficient fear conditioning in psychopathy: A functional magnetic resonance imaging study. American Journal of Psychiatry, 62, 799-805.

Blair, R. J. R. (1995). A cognitive developmental approach to morality: Investigating the psychopath. Cognition, 57, $1-29$.

Blair, R. J. R. (2007). The amygdala and ventromedial prefrontal cortex in morality and psychopathy. Trends in Cognitive Sciences, 11, 387-391.

Buckholtz, J. W., Treadway, M. T., Cowan, R. L., Woodward, N. D., Benning, S. D., Li, R., . . Zald, D. H. (2010). Mesolimbic dopamine reward system hypersensitivity in individuals with psychopathic traits. Nature Neuroscience, 13, 419-421.

Cale, E. M., \& Lilienfeld, S. O. (2002). Sex differences in psychopathy and antisocial personality disorder: A review and integration. Clinical Psychology Review, 22, 1179-1207.

Carré, J. M., Fisher, P. M., Manuck, S. B., \& Hariri, A. R. (in press). Interaction between trait anxiety and trait anger predict amygdala reactive to angry facial expression in men but not women. Social Cognitive and Affective Neuroscience.

Carré, J. M., Gilchrist, J. D., Morrissey, M. D., \& McCormick, C. M. (2010). Motivational and situational factors and the relationship between testosterone dynamics and human aggression during competition. Biological Psychology, 84, 346-353.

Coccaro, E. F., McCloskey, M. S., Fitzgerald, D. A., \& Phan, K. L. (2007). Amygdala and orbitofrontal reactivity to social threat in individuals with impulsive aggression. Biological Psychiatry, 62, 168-178.

Davis, M., \& Whalen, P. J. (2001). The amygdala: Vigilance and emotion. Molecular Psychiatry, 6, 13-34.

Ekman, P., \& Friesen, W. V. (1976). Pictures of facial affect. Palo Alto, CA: Consulting Psychologists.

Etkin, A., Klemenhagen, K. C., Dudman, J. T., Rogan, M. T., Hen, R., Kandel, E. R., \& Hirsch, J. (2004). Individual differences in trait anxiety predict the response of the basolateral amygdala to unconsciously processed fearful faces. Neuron, 44, 1043-1055.

Finger, E. C., Marsh, A. A., Blair, K. S., Reid, M. E., Sims, C., Ng, P., ... Blair, R. J. (2011). Disrupted reinforcement signaling in the orbitofrontal cortex and caudate in youths with conduct disorder or oppositional defiant disorder and a high level of psychopathic traits. American Journal of Psychiatry, 168, 152-162.

Finger, E. C., Marsh, A. A., Mitchell, D. G., Reid, M. E., Sims, C., Budhani, S., . . . Blair, J. R. (2008). Abnormal ventromedial prefrontal cortex function in children with psychopathic traits during reversal learning. Archives of General Psychiatry, 65, 586-594.

First, M. B., Spitzer, R. L., Gibbon, M., \& Williams, J. B. M. (1996). Structured Clinical Interview for DSM-IV Axis I Disorders. New York: New York Biometrics Research, New York State Psychiatric Institute.

Fisher, P. M., Meltzer, C. C., Price, J. C., Coleman, R. L., Ziolko, S. K., Becker, C., ... Hariri, A. R. (2009). Medial prefrontal cortex 5-HT2A density is correlated with amygdala reactivity, response habituation, and functional coupling. Cerebral Cortex, 19, 2499-2507.

Fisher, P. M., Meltzer, C. C., Ziolko, S. K., Price, J. C., Moses-Kolko, E. L., Berga, S. L., \& Hariri, A. R. (2006). Capacity for 5-HT1A-mediated autoregulation predicts amygdala reactivity. Nature Neuroscience, 9,1362-1363.

Forbes, E. E., Brown, S. M., Kimak, M., Ferrell, R. E., Manuck, S. B., \& Hariri, A. R. (2009). Genetic variation in components of dopamine neurotransmission impacts ventral striatal reactivity associated with impulsivity. Molecular Psychiatry, 14, 60-70.

Glenn, A. L., Raine, A., \& Schuq, R. A. (2009). The neural correlates of moral decision-making in psychopathy. Molecular Psychiatry, 14, 5-6.

Gordon, H. L., Baird, A. A., \& End, A. (2004). Functional differences among those high and low on a trait measure of psychopathy. Biological Psychiatry, 56, 516-521.

Guay, J. P., Ruscio, J., Knight, R. A., \& Hare, R. D. (2007). A taxometric analysis of the latent structure of psychopathy: Evidence for dimensionality. Journal of Abnormal Psychology, 116, 701-716.

Hall, J. R., Benning, S. D., \& Patrick, C. J. (2004). Criterion-related validity of the three-factor model of psychopathy personality, behavior, and adaptive functioning. Assessment, 11, 4-16. 
Hare, R. D. (1991). The Hare Psychopathy ChecklistRevised. Toronto: Multi-Health Systems.

Hare, R. D. (1998). Psychopaths and their nature: Implications for the mental health and criminal justice systems. In T. Millon, E. Simonsen, M. Biket-Smith, \& R. D. Davis (Eds.), Psychopathy: Antisocial, criminal and violent behavior (pp. 188-212). New York: Guilford Press.

Hare, R. D. (2006). Psychopathy: A clinical and forensic overview. Psychiatric Clinics of North America, 29, 709-724.

Hare, R. D., \& Neumann, C. S. (2005). Structural models of psychopathy. Current Psychiatry Reports, 7, 57-64.

Hare, R. D., \& Neumann, C. S. (2008). Psychopathy as a clinical and empirical construct. Annual Review of Clinical Psychology, 4, 217-246.

Harenski, C. L., Harenski, K. A., Shane, M. S., \& Kiehl, K. A. (2010). Aberrant neural processing of moral violations in criminal psychopaths. Journal of Abnormal Psychology, 119, 863-874.

Hariri, A. R., Brown, S. M., Williamson, D. E., Flory, J. D., de Wit, H., \& Manuck, S. B. (2006). Preference for immediate over delayed rewards is associated with magnitude of ventral striatal activity. Journal of Neuroscience, 26, 13213-13217.

Hariri, A. R., Drabant, E. M., Munoz, K. E., Bolachana, B. S., Mattay, V. S., \& Egan, M. F. (2005). A susceptibility gene for affective disorders and the response of the human amygdala. Archives of General Psychiatry, 62, 146-152.

Hariri, A. R., Gorka, A., Hyde, L. W., Kimak, M., Halder, I., Ducci, F., . . Manuck, S. B. (2009). Divergent effects of genetic variation in endocannabinoid signaling on human threat- and reward-related brain function. Biological Psychiatry, 66, 9-16.

Hariri, A. R., Mattay, V. S., Tessitore, A., Kolachana, B., Fera, F., Goldman, D., ... Weinberger, D. R. (2002). Serotonin transporter genetic variation and the response of the human amygdala. Science, 297, 400-403.

Hicks, B. M., \& Patrick, C. J. (2006). Psychopathy and negative emotionality: Analyses of suppressor effects reveal distinct relations with emotional distress, fearfulness, and anger-hostility. Journal of Abnormal Psychology, 115, 276-287.

Hill, C. D., Neumann, C. S., \& Rogers, R. (2004). Confirmatory factor analysis of the psychopathy checklist: Screening version in offenders with Axis I disorders. Psychological Assessment, 16, 90-95.

Hyde, L. W., Gorka, A., Manuck, S. B., \& Hariri, A. R. (2011). Perceived social support moderates the link between threat-related amygdala reactivity and trait anxiety. Neuropsychologia, 49, 651-656.

Isen, J., Raine, A., Baker, L., Dawson, M., Bezdjian, S., \& Lozano, D. I. (2010). Sex-specific association between psychopathic traits and electrodermal reactivity in children. Journal of Abnormal Psychology, 119, 216-225.

Jones, A. P., Laurens, K. R., Herba, C. M., Barker, G. J., \& Viding, E. (2009). Amygdala hypoactivity to fearful faces in boys with conduct problems and callous-unemotional traits. American Journal of Psychiatry, 166, 95-102.

Kiehl, K. A., \& Buckholtz, J. W. (2010). Inside the mind of a psychopathy. Scientific American Mind, Sept/Oct, 22-29.
Kiehl, K. A., Smith, A. M., Hare, R. D., Mendrek, A., Forster, B. B., Brink, J., \& Liddle, P. F. (2001). Limbic abnormalities in affective processing by criminal psychopaths as revealed by functional magnetic resonance imaging. Biological Psychiatry, 50, 677-684.

Koenigs, M., Baskin-Sommers, A., Zeier, J., \& Newman, J. P. (2011). Investigating the neural correlates of psychopathy: A critical review. Molecular Psychiatry, 16, 792-799.

Laurell, J., Belfrage, H., \& Hellstrom, A. (2010). Facets on the psychopathy checklist screening version and instrumental violence in forensic psychiatric patients. Criminal Behaviour and Mental Health, 20, 285-294.

Loney, B. R., Taylor, J., Butler, M. A., \& Iacono, W. G. (2007). Adolescent psychopathy features: 6-Year temporal stability and the prediction of externalizing symptoms during the transition to adulthood. Aggressive Behavior, 33, 242-252.

Mahmut, M. K., Menictas, C., Stevenson, R. J., \& Homewood, J. (2011). Validating the factor structure of the Self-Report Psychopathy Scale in a community sample. Psychological Assessment, 23, 670-678.

Manuck, S. B., Brown, S. M., Forbes, E. E., \& Hariri, A. R. (2007). Temporal stability of individual differences in amygdala reactivity. American Journal of Psychiatry, 164, 1613-1614.

Manuck, S. B., Marsland, A. L., Flory, J. D., Gorka, A., Ferrell, R. E., \& Hariri, A. R. (2010). Salivary testosterone and a trinucleotide (CAG) length polymorphism in the androgen receptor predict amygdala reactivity in men. Psychoneuroendocrinology, 35, 94-104.

Marsh, A. A., Finger, E. C., Mitchell, D. G. V., Reid, M. E., Sims, C., Kosson, D. S., ... Blair, R. J. R. (2008). Reduced amygdala response to fearful expression in children and adolescents with callous-unemotional traits and disruptive behavior disorders. American Journal of Psychiatry, 165, 712-720.

Muthen, L. K., \& Muthen, B. O. (2010). Mplus user's guide. Los Angeles, CA.

Neumann, C. S., \& Hare, R. D. (2008). Psychopathic traits in a large community sample: Links to violence, alcohol use, and intelligence. Journal of Consulting and Clinical Psychology, 76, 893-899.

Neumann, C. S., \& Pardini, D. (in press). Factor structure and construct validity of the Self- Report Psychopathy (SRP) Scale and the Youth Psychopathic Traits Inventory (YPI) in young men. Journal of Personality Disorders.

Neumann, C. S., Uzieblo, K., Grombez, G., \& Hare, R. D. (in press). Understanding the Psychopathic Personality Inventory (PPI) in terms of the unidimensionality, orthogonality, and construct validity of PPI-I and -II. Personality disorders: Theory, research, and treatment.

Nikolova, Y. S., Bogdan, R., Brigidi, B. D., \& Hariri, A. R. (2012). Ventral striatum reactivity to reward and recent life stress interact to predict positive affect. Biological Psychiatry, 72, 157-163.

Nouvion, S. O., Cherek, D. R., Lane, S. D., Tcheremissini, O. V., \& Lieving, L. M. (2007). Human proactive aggression: Association with personality disorders and psychopathy. Aggressive Behavior, 33, 552-562.

O'Leary, M. M., Loney, B. R., \& Eckel, L. A. (2007). Gender differences in the association between psychopathic personality traits and cortisol response to induced stress. Psychoneuroendocrinology, 32, 183-191. 
Patton, J. H., Stanford, M. S., \& Barratt, E. S. (1995). Factor structure of the Barratt impulsivity scale. Journal of Clinical Psychology, 51, 768-774.

Paulhus, D. L., Neumann, C. S., \& Hare, R. D. (in press). Manual for the Self-Report Psychopathy Scale (4th ed.). Toronto: Multi-Health Systems.

Paulhus, D. L., Robins, R. W., Trzesniewski, K. H., \& Tracy, J. L. (2004). Two replicable suppressor situations in personality research. Multivariate Behavioral Research, 39, 303-328.

Reuter, J., Raedler, T., Rose, M., Hand, I., Gläscher, J., \& Büchel, C. (2005). Pathological gambling is linked to reduced activation of the mesolimbic reward system. Nature Neuroscience, 8, 147-148.

Rilling, J. K., Glenn, A. L., Jairam, M. R., Pagnoni, G., Goldsmith, D. R., Elfenbein, H. A., \& Lilienfeld, S. O. (2007). Neural correlates of social cooperation and noncooperation as a function of psychopathy. Biological Psychiatry, 61, 1260-1271.

Seara-Cardoso, A., Neumann, C. S., Roiser, J., McCrory, E., \& Viding, E. (2012). Investigating associations between empathy, morality and psychopathic personality traits in the general population. Personality and Individual Differences, 52, 67-71.

Tye, K. M., Prakash, R., Kim, S., Fenno, L. E., Grosenick, L., Zarabi, H., . . . Deisseroth, K. (2011). Amygdala circuitry mediating reversible and bidirectional control of anxiety. Nature, 471, 358-362.

Viding, E., Frick, P. J., \& Plomin, R. (2007). Aetiology of the relationship between callous-unemotional traits and conduct problems in childhood. British Journal of Psychiatry, 190, 33-38.

Vitacco, M. J., Neumann, C. S., Caldwell, M., Leistico, A. M., \& Van Rybroek, G. (2006). The four-factor model of adolescent psychopathy and its association with instrumental violence. Journal of Personality Assessment, 87, 74-83.

Viviani, R. (2010). Unbiased ROI selection in neuroimaging studies of individual differences. Neuroimage, 50, 184-189.

Watt, B., \& Brooks, N. (2011). Self-report of psychopathy in an Australian community sample. Psychiatry, Psychology, and Law, 19, 1-13.

Williams, K. M., Paulhus, D. L., \& Hare, R. D. (2007). Capturing the four-factor structure of psychopathy in college students via self-report. Journal of Personality Assessment, 88, 205-219.

Zhou, Z., Zhu, G., Hariri, A. R., Enoch, M., Scott, D., Sinha, R., ... Goldman, D. (2008). Genetic variation in human NPY expression affects stress response and emotion. Nature, 452, 997-1001. 